\title{
Assessment of EEG Connectivity Patterns in Mild Cognitive Impairment Using Phase Slope Index
}

\author{
Carlos Gómez, Senior Member, IEEE, Saúl J. Ruiz-Gómez, Jesús Poza, Senior Member, IEEE, \\ Aarón Maturana-Candelas, Pablo Núñez, Nádia Pinto, Miguel A. Tola-Arribas, \\ Mónica Cano, and Roberto Hornero, Senior Member, IEEE
}

\begin{abstract}
Mild cognitive impairment (MCI) is a pathology characterized by an abnormal cognitive state. MCI patients are considered to be at high risk for developing dementia. The aim of this study is to characterize the changes that MCI causes in the patterns of brain information flow. For this purpose, spontaneous EEG activity from 41 MCI patients and 37 healthy controls was analyzed by means of an effective connectivity measure: the phase slope index (PSI). Our results showed statistically significant decreases in $P S I$ values mainly at delta and alpha frequency bands for MCI patients, compared to the control group. These abnormal patterns may be due to the structural changes in the brain suffered by patients: decreased hippocampal volume, atrophy of the medial temporal lobe, or loss of gray matter volume. This study suggests the usefulness of $P S I$ to provide further insights into the underlying brain dynamics associated with MCI.
\end{abstract}

\section{INTRODUCTION}

Mild cognitive impairment (MCI) is a pathology characterized by a cognitive deficit higher than expected for a given age and educational level, but insufficient to meet the dementia criteria [1]. Although the cognitive activity of MCI patients is impaired, they maintain their independence in functional and social skills. Recent studies have estimated that the MCI prevalence is between 12 and $18 \%$ for subjects over 60 years [2]. Some of them remain stable over time, but more than $50 \%$ progress to different types of dementia such as dementia due to Alzheimer's disease (AD) [3], vascular dementia [4], Parkinson disease [5], or dementia with Lewy bodies [6]. In particular, the rate of conversion to dementia

This research was supported by 'Ministerio de Economía y Competitividad' and 'European Regional Development Fund' under project TEC2014-53196-R, by 'European Commission' and 'European Regional Development Fund' under project 'Análisis y correlación entre el genoma completo y la actividad cerebral para la ayuda en el diagnóstico de la enfermedad de Alzheimer' ('Cooperation Programme Interreg V-A SpainPortugal POCTEP 2014-2020'), and by 'Consejería de Educación de la Junta de Castilla y León' under project VA037U16. P. Núñez and S. J. Ruiz are in receipt of predoctoral grants co-financed by the 'Junta de Castilla y León' and ESF. N. Pinto's work is partially financed through the FCT postdoctoral grant SFRH/BPD/97414/2013 and projects POCI-01-0145FEDER-007274 and UID/MAT/00144/2013.

C. Gómez, Saúl J. Ruiz-Gómez, J. Poza, A. Maturana-Candelas, P. Núñez, and R. Hornero are with the Biomedical Engineering Group, E.T.S. Ingenieros de Telecomunicación, University of Valladolid, Paseo Belén 15, 47011 Valladolid, Spain (e-mail: carlos.gomez@tel.uva.es).

N. Pinto is with the Institute of Molecular Pathology and Immunology of the University of Porto (IPATIMUP), the Institute for Research and Innovation in Health Sciences, and the Center of Mathematics of University of Porto, Portugal.

M. A. Tola is with the Department of Neurology, Hospital Universitario Río Hortega, Valladolid, Spain.

M. Cano is with the Department of Clinical Neurophysiology, Hospital Universitario Río Hortega, Valladolid, Spain. due to $\mathrm{AD}$ is approximately $15 \%$ per year [3]. Additionally, the medication used to delay symptoms is more effective in the early stages of dementia. For both reasons, an AD early diagnosis is mandatory.

Electroencephalography (EEG) is a non-invasive technique that allows recording the electric fields generated by the brain. EEG is widely used due to its portability, availability and low cost. In addition, EEG has already shown its usefulness to characterize neuronal dynamics in MCI and $\mathrm{AD}[7,8]$.

Brain activity from MCI and AD patients has been widely analyzed with spectral techniques and nonlinear methods. Spectral techniques revealed a slowing of the EEG activity [9], whereas nonlinear methods showed a complexity decrease and a regularity increase of the spontaneous brain activity [10]. However, all these methods measure local activation at the sensor level without taking into account the possible interaction between the different EEG channels. Stam and van Straaten [11] suggested that simple activation studies are not enough to provide a complete characterization of brain dynamics. For this reason, connectivity analysis has focused a great attention in recent years. Previous studies showed lower connectivity values between EEG channels in patients with MCI using Pearson Correlation Coefficient, Magnitude Squared Coherence (MSCOH), and Phase Lag Index $(P L I)[12,13]$. All these measures estimate functional connectivity (i.e. the dependence between remote neurophysiological events). However, it is essential to know the directionality of the relationships to characterize completely the dynamics of the information flow in the brain network. To overcome these limitations, measures of effective or causal connectivity are needed (i.e. the influence that one neuronal system exerts on another), such as Granger Causality $(G C)$, Transfer Entropy $(T E)$, or Phase Slope Index $(P S I)$. The advantage of $P S I$ over other measures of effective connectivity, such as $G C$ and $T E$, lies in its insensitive to false interactions due to volume conduction [14]. The aim of this work is to analyze the EEG connectivity patterns in MCI patients and in healthy controls (HC) by means of an effective connectivity measure $(P S I)$.

\section{MATERIALS AND METHODS}

\section{A. Subjects}

Spontaneous EEG activity was analyzed in $41 \mathrm{MCI}$ patients (17 men and 24 women, age $=76.1 \pm 6.8$ years, mean \pm standard deviation) and $37 \mathrm{HC}$ subjects (12 men and 25 women, age $=76.3 \pm 3.8$ years). No significant differences between groups were found in age ( $p$-value $>0.05$, KruskalWallis test) nor in gender ( $p$-value $>0.05$, Chi-square test). 
TABLE I. SOCIO-DEMOGRAPHIC AND CLINICAL DATA FOR EACH GROUP

\begin{tabular}{|c|c|c|}
\hline & HC group & MCI group \\
\hline Number of subjects & 37 & 41 \\
\hline Age (years) $($ Mean \pm SD) & $76.3 \pm 3.8$ & $76.1 \pm 6.8$ \\
\hline Gender (Male:Female) & $12: 25$ & $17: 24$ \\
\hline Education level $(A: B)^{a}$ & $11: 26$ & $29: 12$ \\
\hline $\operatorname{MMSE}^{\mathrm{b}}\left(\right.$ Mean $\left.^{\prime} \mathrm{SD}\right)$ & $28.8 \pm 1.1$ & $27.2 \pm 1.7$ \\
\hline B-ADL ${ }^{\mathbf{c}}($ Mean \pm SD $)$ & $1.2 \pm 0.2$ & $3.0 \pm 0.9$ \\
\hline
\end{tabular}

Mini-Mental State Examination test (MMSE) was used to evaluate the cognitive deficit of the subjects, while the functional status was assessed by means of the BayerActivities of Daily Living scale (B-ADL). None of the participants in the study was taking any medication that could influence on the EEG data. Table 1 shows the sociodemographic and clinical data for each group.

All participants and patients' caregivers were informed about the research background and the study protocol. Moreover, all of them gave their written informed consent to be included in the study. The Ethics Committee at the "Hospital Universitario Río Hortega" (Valladolid, Spain) endorsed the study protocol, according to The Code of Ethics of the World Medical Association (Declaration of Helsinki).

\section{B. EEG recording}

EEG activity was recorded with a 19-channel equipment $\left(\right.$ XLTEK $^{\circledR}$, Natus Medical, Pleasanton, CA). The subjects were relaxed, awake and with their eyes closed during the EEG acquisition. EEG signals were acquired at a sampling frequency of $200 \mathrm{~Hz}$ for the following 19 electrodes placed following the International System 10-20: Fp1, Fp2, Fz, F3, F4, F7, F8, Cz, C3, C4, T3, T4, T5, T6, Pz, P3, P4, O1 and O2. Independent Component Analysis (ICA) was then applied to minimize the presence of oculographic, cardiographic and myographic artifacts. Afterwards, signals were digitally processed with a FIR filter with a Hamming window between 1 and $70 \mathrm{~Hz}$ and with a notch filter at 50 Hz. Finally, artifact-free epochs of 5-s length (1000 samples) were selected by visual inspection.

\section{Phase Slope Index (PLI)}

PSI is method proposed by Nolte et al. [14] that allows estimating the direction of the information flux. This measure is based on the slope of the phase of cross-spectra between two time series. The cross-spectra for two EEG channels $x_{i}(t)$ and $x_{j}(t)$ is defined as:

$$
S_{i j}(j)=\left\langle\hat{x}_{i}(f) \hat{x}_{j}^{*}(f)\right\rangle,
$$

where $\langle\cdot\rangle$ denotes expectation value and $\hat{x}_{i}(f)$ denotes the Fourier transform for the time series $x_{i}(t)$.

PSI is based on the delay associated to the cause-effect relationship between the times series corresponding to two EEG channels. Assuming that the interaction between these two signals is merely a delay by time, $x_{j}(t)$ can be expressed as:

$$
x_{j}(t)=c x_{i}(t-\tau)
$$

where $c$ is a constant. For the cross-spectra between the two EEG channels:

$$
S_{i j}(f)=\left\langle\hat{x}_{i}(f) \hat{x}_{j}^{*}(f)\right\rangle \propto e^{i 2 \pi f \tau} \equiv e^{i \Phi(f)} .
$$

Equation (3) shows that the phase-spectrum $\Phi(f)=2 \pi f \tau$ is linear and proportional to the time delay $\tau$. Therefore, if the slope of $\Phi(f)$ is positive, the causal direction is estimated to go from $x_{i}(t)$ to $x_{j}(t)$. On the other hand, a negative slope of $\Phi(f)$ indicates that the information flow goes from $x_{j}(t)$ to $x_{i}(t)$.

Taking into account additional requirements, as statistical robustness and insensitivity to signals that do not interact, the PSI between two time series $x_{i}(t)$ and $x_{j}(t)$ can be defined as [14]:

$$
\widetilde{\Psi}_{i j}=\mathfrak{I}\left(\sum_{f \in F} \operatorname{Coh}_{i j}^{*}(f) \operatorname{Coh}(f+\delta f)\right),
$$

where $\operatorname{Coh}_{i j}(f)=S_{i j}(f) / \sqrt{S_{i i}(f) S_{j j}(f)}$ is the complex coherency, $S_{i j}(f)$ is the cross-spectral matrix, $\delta f$ is the frequency resolution at a given frequency band $F$, and $\mathfrak{J}(\cdot)$ denotes the imaginary part.

Finally, it is convenient to normalize $\widetilde{\Psi}$ by an estimate of its standard deviation:

$$
\Psi=\widetilde{\Psi} / \operatorname{std}(\widetilde{\Psi}),
$$

with $\operatorname{std}(\widetilde{\Psi})$ being estimated using jackknife method [14]. $P S I$ values around zero must be ignored in order to improve the robustness and reliability of the method [15].

\section{RESULTS}

PSI results were calculated at the EEG typical frequency bands: $\delta(1-4 \mathrm{~Hz}), \theta(4-8 \mathrm{~Hz}), \alpha(8-13 \mathrm{~Hz}), \beta 1(13-19 \mathrm{~Hz}), \beta 2$ $(19-30 \mathrm{~Hz})$, and $\gamma(30-70 \mathrm{~Hz})$. Figure 1 shows the mean PSI values for $\mathrm{MCI}$ and $\mathrm{HC}$ groups at the connections that showed statistically significant differences between both groups. The third column of Figure 1 illustrates the corresponding $p$-values (Mann-Whitney $U$ test): red color tones indicate significant increases in connectivity in MCI patients compared to $\mathrm{HC}$ subjects, while blue color tones indicate significant decreases in MCI group in comparison with $\mathrm{HC}$ one. Our results revealed that EEG activity in MCI patients is characterized by a decrease in effective connectivity, mainly at $\delta$ and $\alpha$ bands. Specifically, PSI values show a significant decrease of the information flow from the lateral brain regions to the frontal area in $\delta$ and $\gamma$ bands, from the frontal area to the posterior one in the $\theta$ band, and from central and posterior regions to the left lateral area in $\delta$ and $\alpha$ frequency bands.

Receiver operating characteristic (ROC) curves with a leave-one-out cross-validation procedure were used to assess the ability of PSI to discriminate MCI patients form $\mathrm{HC}$ subjects. The highest accuracy value was obtained for the connection $\mathrm{T} 6$ to $\mathrm{Fp} 2$ at $\delta$ band: $71.79 \%$ (sensitivity of $78.38 \%$, specificity of $65.85 \%$, and area under the ROC curve of 0.71 ). 


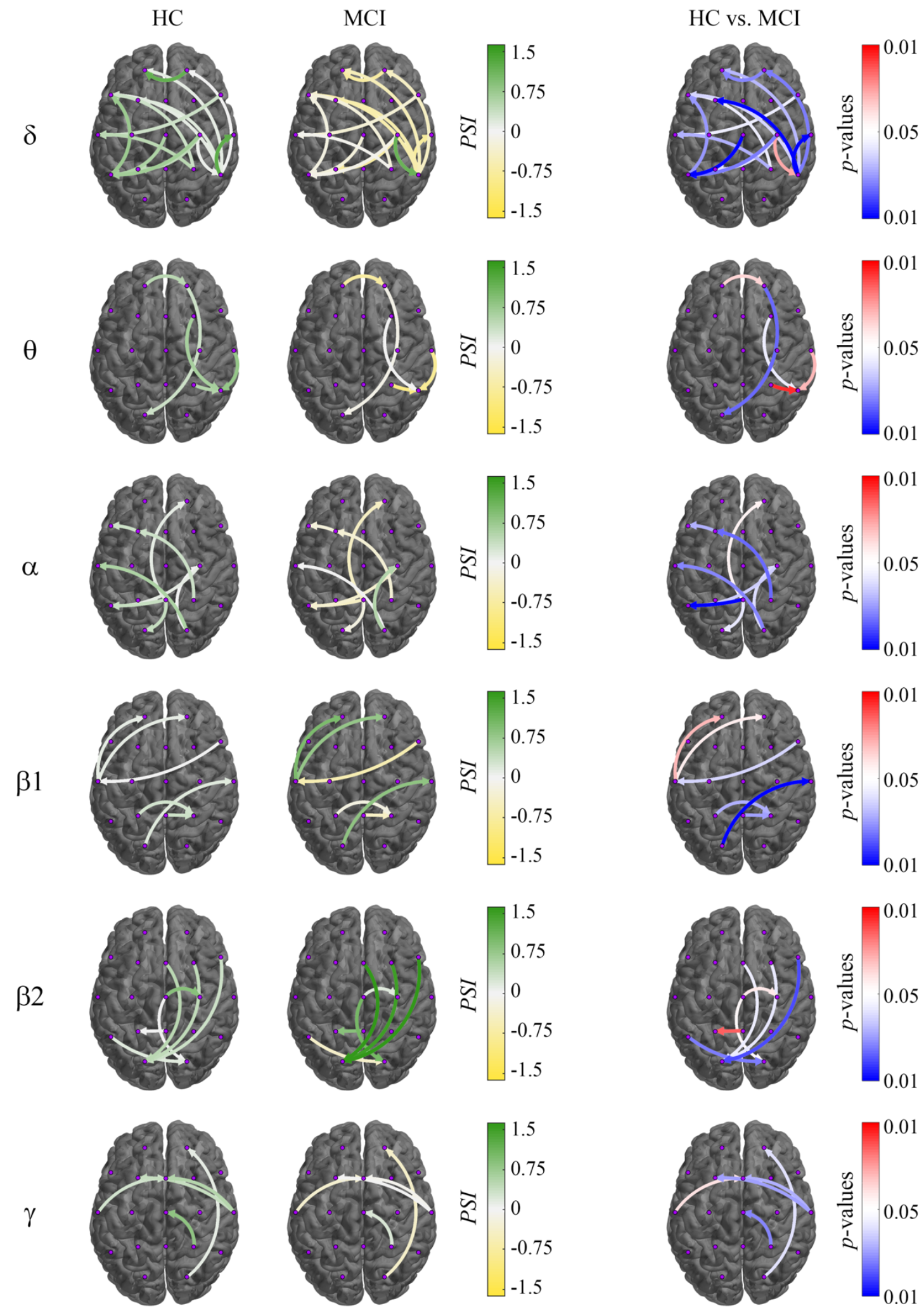

Figure 1. Connectivity patterns obtained with PSI for HC (left column) and MCI (central column) groups, where the arrows indicate the direction of the information flow. Right column shows the statistically significant differences between groups: red color tones indicate significant increases in connectivity in MCI patients compared to $\mathrm{HC}$ subjects, while blue color tones indicate decreases. Connections were only displayed when statistically significant differences between groups were found ( $p$-values $<0.05$, Mann-Whitney $U$ test). 


\section{DISCUSSION AND CONCLUSIONS}

In this study, spontaneous EEG activity was analyzed from $41 \mathrm{MCI}$ patients and $37 \mathrm{HC}$ subjects using PSI, a measure of effective connectivity. Our results show that PSI values are significantly lower for patients with MCI at the low frequency bands. Causal measures have been previously applied to EEG and functional magnetic resonance imaging (fMRI) data, revealing diminished connectivity between different brain areas [16,17]. Additionally, our PSI results showed an alteration of the information flow patterns in all the frequency bands, being more remarkable for long distance connections and for low frequency bands. These results agree with previous studies that reported a connectivity decrease between the frontal region and the lateral and posterior areas in the $\delta$ and $\theta$ bands using functional measures, such as $P L I$ [12]. Causal measures, as $G C$ and Directed Transfer Function $(D T F)$, also revealed a connectivity decrease between the frontal and posterior areas, although the affected frequency bands differ $[13,18]$. This inconsistency on the results may be due to the heterogeneity of $\mathrm{MCI}$, to the different neuroimaging techniques, to the different connectivity coupling measures, or to a combination of these factors. Clinically, the observed abnormal patterns may be due to the structural brain changes suffered by patients with MCI: decreased hippocampal volume, atrophy of the medial temporal lobe, or loss of gray matter volume [19].

Finally, an accuracy of $71.79 \%$ was achieved using a ROC curve with a leave-one-out cross-validation procedure. Previous studies reported accuracies between $69.8 \%$ and $83 \%$ for $\mathrm{HC}$ vs. MCI classification using different coupling measures $[13,16,20]$. For instance, accuracy values of $69.8 \%$ were reached when $\mathrm{MSCOH}$ and synchronization likelihood were applied to magnetoencephalographic (MEG) recordings [20]. Dauwels et al. [16] yielded classification rates of $83 \%$ using $D T F$. Finally, $G C$ method correctly classified $79.5 \%$ of the $\mathrm{MCI}$ and $\mathrm{HC}$ subjects who participated in a recent study.

It is important to mention some limitations of this research work. First, it would be convenient to increase the sample size to augment the statistical power of the results. Second, we have analyzed all the possible combinations between the different EEG electrodes in the current study; it would be interesting to divide the brain into regions to try to facilitate the interpretation of the results in spite of losing spatial resolution. Finally, it would be interesting to use other measures of effective connectivity, such as $G C, D T F$, or $T E$, to characterize brain information flow patterns from different perspectives.

We can conclude that the analysis of EEG activity using PSI method revealed that MCI causes significant changes in the brain activity. This study constitutes an initial step to demonstrate the possible application of novel measures of effective connectivity, such as PSI, for the characterization of $\mathrm{AD}$ in early stages.

\section{REFERENCES}

[1] B. Winblad, K. Palmer, M. Kivipelto, V. Jelic, L. Fratiglioni, L.O. Wahlund, et al., "Mild cognitive impairment - Beyond controversies, towards a consensus: Report of the International Working Group on Mild Cognitive Impairment," J. Intern. Med., vol. 256, pp. 240-246, 2004.

[2] RC. Petersen, "Mild cognitive impairment," Contin. (Minneap. Minn.), vol. 22, pp. 404-418, 2016.

[3] C. Davatzikos, P. Bhatt, L.M. Shaw, K.N. Batmanghelich, and J.Q. Trojanowski, "Prediction of MCI to AD conversion, via MRI, CSF biomarkers, and pattern classification," Neurobiol. Aging, vol. 32, pp. $1-17,2011$.

[4] J.S. Meyer, G. Xu, J. Thornby, M.H. Chowdhury, and M. Quach, "Is mild cognitive impairment prodromal for vascular dementia like Alzheimer's disease?," Stroke, vol. 33, pp. 1981-1985, 2002.

[5] C.C. Janvin, J.P. Larsen, D. Aarsland, and K. Hugdahl, "Subtypes of mild cognitive impairment in Parkinson's disease: Progression to dementia," Mov. Disord., vol. 21, pp. 1343-1349, 2006.

[6] J. Molano, B. Boeve, T. Ferman, G. Smith, J. Parisi, D. Dickson, et al., "Mild cognitive impairment associated with limbic and neocortical Lewy body disease: A clinicopathological study," Brain, vol. 133, pp. 540-556, 2010.

[7] J. Dauwels, F. Vialatte, and A. Cichocki, "Diagnosis of Alzheimer's disease from EEG signals: where are we standing?," Curr. Alzheimer Res., vol. 7, pp. 487-505, 2010.

[8] J. Jeong, "EEG dynamics in patients with Alzheimer's disease," Clin. Neurophysiol., vol. 115, pp. 1490-1505, 2004.

[9] M. Baker, K. Akrofi, R. Schiffer, and M.W. O'Boyle, "EEG patterns in mild cognitive impairment patients," Open Neuroimag. J., vol. 2, pp. 52-55. 2008.

[10] C.J. Stam, "Nonlinear dynamical analysis of EEG and MEG: Review of an emerging field," Clin. Neurophysiol., vol. 116, pp. 2266-2301, 2005.

[11] C.J. Stam and E.C.W. van Straaten, "The organization of physiological brain networks," Clin. Neurophysiol., vol. 123, pp. 1067-1087, 2012

[12] B. Tóth, B. File, R. Boha, Z. Kardos, Z. Hidasi, Z.A. Gaál, et al., "EEG network connectivity changes in mild cognitive impairment Preliminary results," Int. J. Psychophysiol., vol. 92, pp. 1-7, 2014.

[13] C. Gómez, C. Juan-Cruz, J. Poza, S.J. Ruiz-Gómez, J. Gomez-Pilar, P Núñez, et al., "Alterations of effective connectivity patterns in mild cognitive impairment: An MEG study," J. Alzheimer's Dis. In Press, 2018.

[14] G. Nolte, A. Ziehe, V.V. Nikulin, A. Schlögl, N. Krämer, T. Brismar, and K.R. Müller, "Robustly estimating the flow direction of information in complex physical systems," Phys. Rev. Lett., vol. 100, pp. 1-5, 2008.

[15] A. Ewald, F.S. Avarvand, and G. Nolte, "Identifying causal networks of neuronal sources from EEG/MEG data with the phase slope index: a simulation study," Biomed. Tech. (Berl.), vol. 58, pp. 165-178, 2013.

[16] J. Dauwels, F. Vialatte, F. Musha, and A. Cichocki, "A comparative study of synchrony measures for the early diagnosis of Alzheimer's disease based on EEG," Neuroimage, vol. 49, pp. 668-693, 2010.

[17] Z. Liu, L. Bai, R. Dai, C. Zhong, H. Wang, Y. You, et al., "Exploring the effective connectivity of resting state networks in mild cognitive impairment: an fMRI study combining ICA and mGCA," Conf. Proc. IEEE Eng. Med. Biol. Soc., pp. 5454-5457, 2012.

[18] F. Vecchio and C. Babiloni, "Direction of information flow in Alzheimer's Disease and MCI patients," Int. J. Alzheimers. Dis., vol. 2011, pp. 1-7, 2011.

[19] G.B. Karas, P. Scheltens, S.A.R.B. Rombouts, P.J. Visser, R.A. Van Schijndel, N.C. Fox, and F. Barkhof, "Global and local gray matter loss in mild cognitive impairment and Alzheimer's disease," Neuroimage, vol. 23, pp. 708-716, 2004.

[20] C. Gómez, C.J. Stam, R. Hornero, A. Fernández, and F. Maestú, "Disturbed beta band functional connectivity in patients with mild cognitive impairment: an MEG study," IEEE Trans. Biomed. Eng., vol. 56, pp. 1683-1690, 2009. 